\title{
Solid-liquid flow model and the impact of turbulent flow field on stirred parameters for oil chili sauce
}

\author{
Liya Yu, Shaobo Li, Lifei Luo*, Ling He \\ School of Guizhou University, Guiyang 550003, China \\ *Corresponding author: Lifei Luo
}

\begin{abstract}
From the study of mixed solid-liquid flow characteristics of viscous liquid, solid-liquid model of two phase flow for oil chili sauce in the stirring apparatus is presented in this paper. The impacts of turbulent flow field for stirred parameters in the stirring apparatus are studied. Combining with the study of oil chili fluid flow characteristics, proposes the solid flow model and turbulent flow field on stirre parameters on oili sauce, for determining of the stirring speed and so on.
\end{abstract}

KEYWORD: Oil chili sauce, Solid-liquid flow model, Stirring device, Turbulence model

\section{INTRODUCTION}

Guizhou condiment is the main of chili. And there are 120 chili varieties in the Guizhou province. It is difficult to store fresh chilli, and dry chili is low addvalue. Therefore, deep processing of chili in creating added value is very important. Oil chili sauce which contains chili, lobster sauce, edible oil, Chinese prickly ash and meat is a high value-added deep-processing of chili. For traditional automatic filling technology, solid-liquid ratio and filling accuracy already can't good meet mixed solid-liquid of oil chili sauce automatic filling requirement.

Mixed proportion and two-phase flow characteristics are the key technology to research the complex solid-liquid mixture filling for oil chili sauce. Further, the research of two-phase flow characteristics in solid-liquid mixing technology is important for filling machine development, and most researchers now focus on mixed characteristics, power consumption and flow characteristics. Liang super (Li Liang chao, 2014) Euler-Euler two-fluid model based on particle dynamics of sinking particles and floating particles of solid-liquid suspension characteristics were studied. Lucy Liu (Liu Yu ling, 2014) on the flow field in a stirred tank was analyzed. R. Angst, M. Kraume (R. Angst, 2006) in a stirred tank with three different power consumption of solid-liquid two-phase mixture was stirred at different concentrations were experimentally study also shows the results of experiments using CFD predictions need to be amended. This paper by drawing on the physical characteristics can be controlled stirring of other pharmaceutical and chemical properties of the reference material, and combined with the physical characteristics of the system itself chili oil, to carry out the relevant theoretical and experimental studies, which made chili oil to ensure optimization of product quality and process stability parameters for the promotion of comprehensive utilization and deep development of pepper is of great importance.

\section{CHILI LIQUID TURBULENT MIXING PLANT OIL SYSTEM MODEL}

\subsection{Basic features mixing process}

According to the views of fluid mechanics, fluid can be divided into two major categories of ideal fluid and the actual fluid. Ideal fluid in the flow without resistance, is called a non-viscous fluid; When the actual fluid flow resistance within the friction (or shear), called a viscous fluid. According to the effect on the fluid shear stress and the shear rate, the relationship between the viscous fluid and can be divided into Newtonian fluids and non-Newtonian fluid. After Newton fluid refers to the mechanical deformation extremely easily, and the shear stress and deformation rate is proportional to the low viscous fluid. All different from the Newtonian fluid are referred to as non-Newtonian fluid. Mixing oil system of chili liquid in the tank is a kind of solid-liquid mixed viscous liquid, obviously is a Newtonian fluid. Stirring can make two or more different material dispersed to each other, so as to achieve uniform mixing, also can accelerate the heat transfer and mass transfer process. 


\subsection{Mixing oil groove system chili liquid turbulence model}

Mixing device in the main flow characteristic of fluid is turbulent, In theory, all turbulence can be full $\mathrm{Na}$ vier-Stokes equations are solved numerically solving [4], conservation in the form of unsteady three-dimensional Renault (Reynolds) average Navier-Stokes equations as follows:

Continuous equation:

$\frac{\partial \rho}{\partial t}+\frac{\partial}{\partial x_{i}}\left(\rho u_{i}\right)=0$

The momentum equation:

$\frac{\partial \rho}{\partial t}\left(\rho u_{i}\right)+\frac{\partial}{\partial x_{i}}\left(\rho u_{i} u_{j}\right)=-\frac{\partial P}{\partial x_{i}}+\frac{\partial \tau_{i j}}{\partial x_{j}}$

Energy equation:

$\frac{\partial}{\partial t}(\rho E)+\frac{\partial}{\partial x_{j}}\left(\rho u_{j} H\right)=\frac{\partial P}{\partial t}+\frac{\partial}{\partial x_{j}}\left(u_{i} \tau_{i j}+\lambda \frac{\partial T}{\partial x_{j}}\right)$

In the formula,

$\tau_{i j}=\mu_{t}\left(\frac{\partial u_{i}}{\partial x_{j}}+\frac{\partial u_{j}}{\partial x_{i}}-\frac{2}{3} \delta_{i j} \frac{\partial u_{i}}{\partial x_{j}}\right)$

Among them, $\rho, t, u_{i},{ }^{X_{j}}, P, E, H, T$ respectively, the density, time, velocity components, coordinate geometry, pressure and internal energy, enthalpy and temperature, turbulence viscosity coefficient, respectively, for the turbulent Prandtl constant, Reynolds stress, according to the influence of turbulence.

(1) the turbulence intensity

If mixing between shaft and blade groove for clearance, clearance area full of turbulent boundary layer can be thought of, during which time averaged velocity field distribution as follows:

$$
\left\{\begin{array}{lr}
\bar{u}_{\theta}=\frac{u_{2}}{2 \operatorname{arctg}\left(\frac{\sqrt{R_{e}} \kappa}{2}\right)} \operatorname{arctg}\left(\frac{\sqrt{R_{e}} \kappa\left(r_{1}-r_{2}\right)}{\delta}\right) & \left(r_{1} \leq r \leq r_{1}+\delta / 2\right) \\
\bar{u}_{\theta}=u_{2}+\frac{u_{2}}{2 \operatorname{arctg}\left(\frac{\sqrt{R_{e}} \kappa}{2}\right)} \operatorname{arctg}\left(\frac{\sqrt{R_{e}} \kappa\left(r_{1}+\delta-r\right)}{\delta}\right) & \left(r_{1}+\delta / 2 \leq r \leq r_{1}+\delta\right)
\end{array}\right.
$$

The shear stress field is:

$$
\left\{\begin{array}{cc}
\tau_{r \theta}=\frac{u_{2} \sqrt{R_{e}} \kappa}{2 \delta \operatorname{arctg}\left(\frac{\sqrt{R_{e}} \kappa}{2}\right)} & \left(r_{1} \leq r \leq r_{1}+\delta / 2\right) \\
\tau_{r \theta}=\frac{\mu u_{2} \sqrt{R_{e}} \kappa}{2 \delta \operatorname{arctg}\left(\frac{\sqrt{R_{e}} \kappa}{2}\right)} & \left(r_{1}+\delta / 2 \leq r \leq r_{1}+\delta\right)
\end{array}\right.
$$

Type, $R_{e}$ as the Reynolds number; $\mu$ Is the droplet deformation stress coefficient, equal to the ratio of shear stress and shear rate; $\kappa$ for turbulent Karman constant, desirable $\kappa=0.2 \sim 0.4$, or use to calculate $\kappa=0.125 R_{e}^{0.07} ; u_{2}$ for the blade linear velocity; $r_{1}$ and $r_{2}$ the radius of the rotor and blade respectively.

(2) The Reynolds number

With chili oil system mixing Reynolds number increases, groove groove of the circulation flow pattern also were characterized by laminar flow, transition flow and turbulence, $<50$, groove fluid performance for laminar flow; $50<<104$, groove fluid performance for transition flow; $>104$, groove fluid is turbulent.

\section{CHILI OIL SYSTEM FLUID FORM IMPACT ON MIXING PARAMETERS}

Stirring device need to consider about stirring the material properties, mixing purpose, production requirements, production requirements, demand for power and equipment costs and so on.

\subsection{The mixing mode and shape of the device}

Stirring device can be divided into straight blade paddle, open turbine type, push, disk turbine, anchor, ribbon, spiral and other shapes, depending on the viscosity of different liquids can be divided into a low viscosity fluid mixing device, high viscosity liquid stirring devices.

It is difficult to achieve goals to adopt vertical mixing form because the filling equipment mixing oil groove of chili liquid viscosity is bigger and continuous stirring flow patterns and export more uniform flow material etc.so this paper used impeller type which is Horizontal spiral ribbon, horizontal mixing machine is comprised of a U-shaped trough, ribbon agitator blades and transmission components, as illustrated in Fig. 1.

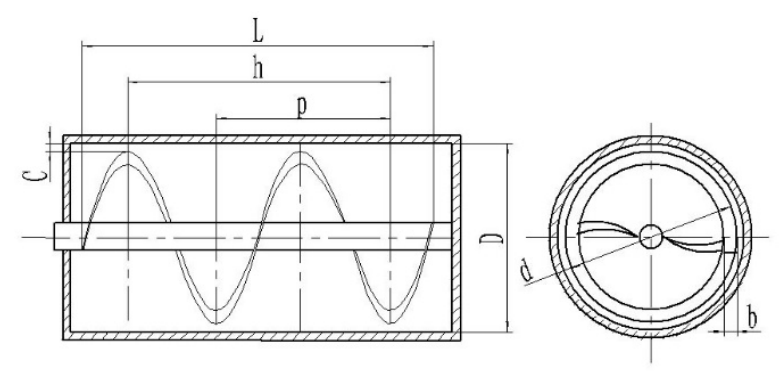

C - blade and tank wall distance; D - agitation tank diameter; B - ribbon width; D - ribbon diameter; $\mathrm{H}$ - ribbon section height; $\mathrm{P}$ - pitch; L mixing plant total height

Fig. 1. Horizontal spiral ribbon agitator

\subsection{Stirring device geometry parameter}

(1) The diameter of the stirring shaft $d_{0}$

Generally should be calculated on torsional deformation, stirring shaft by combination of the torsion and bending moment, torsional deformation will cause the vibration of the state, make oil seal failure, so the shaft on the unit length should be the biggest torsion Angle limit within the scope of the permit,

$$
d_{0} \geq 155.4\left(\frac{M_{n \max }}{[\gamma] G\left(1-N_{0}^{4}\right)}\right)^{\frac{1}{4}}
$$


The type, $[\gamma]$ it is permissible torsion Angle; $M_{\text {nmax }}$ is the maximum torque shaft transmission, if the drive for efficiency $\eta$, then $M_{n \max }=9553 \frac{P}{n_{d}} \eta ; G$ is the shaft material shear modulus of elasticity; N0 the ratio of inner and outer diameters is hollow shaft.

(2) The mixing device blade diameter (screw with diameter) $d$

According to the experiment, the slot size $D$ $=100-600 \mathrm{~mm}$, tub a certain diameter, determines the size of the impeller blade diameter and distance on the surface of the cylinder body wall, namely clearance, generally take $d / D=0.73-0.98$, according to the characteristics of the slurry an material, through mixing plant blade diameter to tank diameter is obtained by mixing blade diameter.

(3) Pitch $p$

Experiment take: $p / d=0.386-2.15$. The radial movement of fluid in the $p / d<0.664$ there is no obvious regularity, when $p / d$ increases to 1.0 , the sharp rise in the radial velocity.

(4) The ribbon width

Experiment take: $b / d=0.0836$ to 0.266 .

(5) The ratio of the wheel and shaft

Round the ratio of impeller diameter and the diameter of shaft called round than, tell from the hydraulic performance, reduce the ratio of the wheel and shaft, can reduce the hydraulic friction loss, increase the mixing push flow area, and is helpful for the improvement of the cavitations resistance.

\subsection{The Power of Stirred Device}

The consumed energy of stirred device is mainly provided to the material in mixing plant to shear and circulation. The mixing power means the energy that is used to put the material into the slurry pool per unit time, during mixing, but the energy that is consumed in the shaft seal and transmission device is excludes. There are 2 aims in calculating the stirring power. One is to design or nuclear the stirred device and the strength and stiffness of the stirring shaft. The other is to select the gearing, such as the motor and transmission. When some stirred device stir a certain physical properties of materials in a specific speed, the power that stirred device work on the material is happening.

\section{CRITICAL SPEED}

The lowest stirring speed that can make solid-liquid two phase flow achieve full state of suspension from the bottom is called critical speed of revolution, $\mathrm{Nja}$, There were a number of critical speed, Nja, of correlations reference about solid-liquid two phase flow. Referencing (Yang Feng ling, 2012) connection type that belongs to Zwietering, blade mixing critical speed can be written as:

$$
N_{j a}=K d^{0.85} v^{0.1} d_{p}^{0.2}\left|g \frac{\rho_{p}-\rho}{\rho}\right|^{0.45}\left|100 \times \frac{\rho_{p} \varphi_{V}}{\rho\left(1-\varphi_{V}\right)}\right|
$$

$\mathrm{N}_{\mathrm{ja}}$ is the critical speed; $\mathrm{K}$ is the equation constant; $\mathrm{v}$ is kinematic viscosity of liquid; $\rho$ is the density of the fluid; $\rho_{p}$ is the density of solid particles; $d_{p}$ is the diameter of the solid; $\varphi$ is the solid volume fraction; $\mathrm{d}$ is the blade diameter; $\mathrm{g}$ is the acceleration of gravity.

\section{THE EXPERIMENTAL ANALYSIS AND CONCLUSION}

According to the above theory, the main parameters could be shown in table 1

Table 1 Main parameters of model (unit $\mathrm{mm}$ ):

\begin{tabular}{lll}
\hline Number & parameters & data \\
\hline 1 & Agitation tanks bore D & 360 \\
2 & Ribbon diameter d & 320 \\
3 & The distance between & 20 \\
4 & ribbon and wall C & 40 \\
5 & Ribbon width b & 800 \\
6 & Ribbon height h & 800 \\
7 & Pitch p & 800 \\
\hline
\end{tabular}

(1)The calculation of power energy in stirring test does not include the energy consumption in shaft seal and transmission device, equipment debugging test show that the consumed power of shaft seal is bigger, is just the $5 \%$ to $10 \%$ of stirring the consumed power. So when choose the stirring, motor the parameters should be reserved.

(2)In order to achieve the mixing uniform state when stir chili liquid, the stirring of chili liquid is firstly requested to provide certain amount of circulation, to avoid dead zones in the stirring tank, and make the spiral ribbon exceeds a certain speed(critical speed), so that chili liquid is stirred achieve rapid convective circular motion; and request the turbulence intensity and shear velocity in the stirred tank is relatively large, the shearing benefit to the dispersion of chili dough in the tank and the strengthening of turbulence expansion. Although higher speed may improve the uniformity of chili liquid in the tank on the overall, it has little effect on improving the filling uniformity, even affect the quality of filling oil chili.

(3)The width of helical ribbon have a great influence on the mixing efficiency, when the width of helical ribbon is doubled (from $\mathrm{b}=20 \mathrm{~mm}$ to $40 \mathrm{~mm}$ ), chili liquid mixing efficiency nearly double and increased significantly.

(4)The Change of screw pitch have little effect on the rate of mixing, when the clearance of helical ribbon and tank wall is doubled within recommended value, the mixed time increased less than $10 \%$. 


\section{ACKNOWLEDGEMENTS}

Project supported by the National Natural Science Foundation of China (No. 51475097), The national science and technology support program of China (No.2012BAF12B14), the project of science and technology of Guizhou Province(No. JZ [2014] 2001 and [2012]6008) and talent introduction research program of Guizhou University (No. (2014)60).

\section{REFERENCE}

Li Liang chao,Xu Bin,Yang Jun.Sinking/Floating Pariticles Solid Suspension Characteristics in Stirred Tank Based on CFD Simulation[J].Journal of mechanical engineering,2014,12:185-191.

Liu Yu ling, Zheng Yan, Wei Wen li . Study on flow characteristics in a stirred tank by large-eddy simulation [J]. Chinese Journal of Computational Mechanics, 2014,05:634-639.

Liu Shi he, Engineering Turbulence, Beijing: Science press, 2011. 1

R. Angst, M. Kraume. Experimental investigations of stirred solid/liquid systems in three different scales:Particle distribution and power consumption[J], Chemical Engineering Science, 2006,61:2864-2870

Yang Feng ling, Zhou Shen Jie, Zhang Cui xun, Wang Gui chao. Solid-liquid Suspension in an Unbaffied Stirred Tank [J]. Journal of Sichuan University (Engineering Science Edition),2012,04:185-190. 